was used in the following departments: ICU (74\%), Surgery $(7 \%)$ Internal Medicine (5\%), and other several wards (14\%). Colistin treatment was started empirically in $16 \%$ of patients. Microbiological diagnosis (Pseudomonas sp. that were aminoglycosides and carbapenems-resistant, and multi-drug resistant Acinetobacter baumannii) was the reason for Colistin treatment in $84 \%$ of patients.

Conclusions Due to the increased number of patients in a seriously difficult, life-threatening conditions caused by severe nosocomial infections it is necessary to establish strict control over Colistin prescribing (an antibiogram based on blood culture or cerebrospinal fluid, council of infectiology experts etc.). The possibility of getting it registered in Serbia and included on the list of reimbursed drugs should be investigated. It is also necessary to monitor carefully, and to improve our active communication with, the main wards in order to promote the rational use of antibiotics.

No conflict of interest.

\section{OHP-073 SURVEY OF INTERFACE MANAGEMENT MEASURES REGARDING MEDICINES}

doi:10.1136/ejhpharm-2013-000276.446

'S Vogler, ' $\mathrm{N}$ Zimmermann, ${ }^{1} \mathrm{C}$ Habl, ' $\mathrm{C}$ Leopold, ${ }^{2} \mathrm{E}$ Dolinar. 'Gesundheit Österreich GmbH/Austrian Health Institute, Health Economics, Vienna, Austria; ${ }^{2}$ Retired, Chief Hospital Pharmacist, Vienna, Austria

Background The need to improve medicines management at the interface of hospital and primary care is generally acknowledged. But knowledge of good practise on how to bridge that gap is scant.

Purpose To learn about existing policies, mechanisms and measures of cooperation between the hospital and primary sector (hereafter called interface management).

Materials and Methods A survey was performed with the PHIS (Pharmaceutical Pricing and Reimbursement Information) network comprising competent authorities for pharmaceutical pricing and reimbursement as well as hospital pharmacists from 27 countries (25 EU Member States, Norway and Turkey). PHIS network members were asked to inform in writing, preferably by drafting a report according to a predefined template, of medicines management in the in-patient sector and interface management measures in their country. We reviewed 19 published PHIS Hospital Pharma reports, two draught reports and information provided by six further countries (data as of 2009/2010). During a network meeting in February 2012, network members from eleven countries provided updated information on interface management measures in their country on a poster.

Results Only 17 countries reported interface management initiatives. Measures included joint reimbursement lists, hospital drug formularies being coordinated with the list of recommendations for medicines in the primary care, joint development of recommendations/guidelines; joint Drugs and Therapeutics Committees (DTC) and hospital DTCs with a representative from the social health insurance; (obligatory) transfer of information on pharmacotherapy between the sectors, including IT solutions; patient education and counselling; special funding schemes, financial incentives for cooperation projects; pharmacy liaison services, hospital discharge programmes and medicines reconciliation.

Conclusions As in most cases the implementation of the reported measures would require a change in the organisation and funding of the pharmaceutical system, it cannot be done by the hospital pharmacists alone. Improved dialogue between the sectors is urgently needed.

No conflict of interest.

\section{OHP-074 THE CHRONIC OBSTRUCTIVE PULMONARY DISEASE IN AZIENDA SANITARIA PROVINCIALE SIRACUSA: ECONOMIC CONSIDERATIONS RELATED APPROPRIATENESS OF PRESCRIPTION}

doi:10.1136/ejhpharm-2013-000276.447

'N Avola, ${ }^{2} \mathrm{~S}$ Guzzardi, ${ }^{2} \mathrm{D}$ Sgarlata, ${ }^{2} \mathrm{R}$ Sorbello, ${ }^{2} \mathrm{G}$ Cacciaguerra, ${ }^{2} \mathrm{~A}$ Scandurra. 'Università degli Studi di Catania, Scuola di Specializzazione Farmacia Ospedaliera, Catania, Italy; ${ }^{2}$ P.O. Umberto I, U.O.C. Farmacia, Siracusa, Italy

Background In Italy respiratory diseases are the third cause of death, $50 \%$ of which is caused by Chronic Obstructive Pulmonary Disease (COPD). COPD is an irreversible inflammation that causes narrowing of the airways and has a slow and progressive course. In Siracusa the high incidence of COPD may be due to the petrochemical plants in the area. Drug treatment allows us to improve quality of life and to reduce mortality, but often the prescriptions do not adhere to the GOLD Guidelines (GL) for COPD treatment.

Purpose To assess the budgetary impact of the treatment used and of the GOLD GL treatment.

Materials and Methods The authors obtained, by administrative databases and mathematical models:

- The prescriptions of medicines for COPD (ATC R03) in 2010 in Azienda Sanitaria Provinciale (ASP) Siracusa;

- The number of patients with COPD;

- The number of patients for each stage of severity;

- The budget impact of the treatment used and the GOLD GL treatment, which recommends:

- using SAMAs/SABAs(short-action antimuscarinics/antiadrenergics) in the mild stage;

- adding LAMAs/LABAs(long-action antimuscarinics/antiadrenergics) from the moderate to very severe stage;

- to add FDCs (fixed combination drugs)/ICSs (inhaled corticosteroids) in severe and very severe stages.

Results 5895 patients had COPD, of whom:

- 1484 in mild stage;

- 2672 in moderate stage;

- 1155 in severe stage;

- 584 in very severe stage.

The spending for drugs prescribed for COPD was $€ 2,702,627$ of which $€ 1,787,967$ was for FDCs/ICSs.

If the prescriptions were $100 \%$ adherent to GOLD GL spending would have been $€ 1,309,304$, of which $€ 434,029$ for FDCs/ICSs, with a saving of $€ 1,393,323$.

Conclusions If the prescriptions of FDCs/ICSs adhered to GOLD GL, spending would have been $50 \%$ less. The adherence to GOLD GL ensures the patient a proper prescription and allows high savings. The authors are developing a training-information project aimed at encouraging doctors to prescribe appropriately.

No conflict of interest.

\section{OHP-075 THE COST OF MANAGING INTRACRANIAL ANEURYSMS BY EMBOLIZATION IN MOROCCO}

doi:10.1136/ejhpharm-2013-000276.448

${ }^{1} \mathrm{~A}$ Cheikh, ${ }^{2} \mathrm{~S}$ Ahid, ${ }^{1} \mathrm{~N}$ El Abbadi, ${ }^{1} \mathrm{H}$ Ismaili, ${ }^{1} \mathrm{~A}$ Ababou, ${ }^{2} \mathrm{Y}$ Cherrah, ${ }^{1} \mathrm{~A}$ El Quessar 'Mohammed $V$ souissi university, Faculty of medecine and pharmacy and CHEIKH ZAID hospital, Rabat, Morocco; 'Mohammed V souissi university, Faculty of medecine and pharmacy, Rabat, Morocco

Background The overall prevalence of intracranial aneurysm is thought to be between 0.5 to $6 \%$ of population, based on angiographic study and autopsies. The frequency of detection and treatment of these aneurysms has increased due to the greater use of non-invasive diagnostic imaging techniques. 